\title{
Parasitas em ostras de cultivo (Crassostrea rhizophorae e Crassostrea gigas) da Ponta do Sambaqui, Florianópolis, SC
}

\author{
[Parasites in cultured oysters (Crassostrea rhizophorae and Crassostrea gigas) from \\ Ponta do Sambaqui, Florianópolis, SC] \\ R.C. Sabry, A R.M. Magalhães \\ Centro de Ciências Agrárias - Departamento de Aqüicultura da Universidade Federal de Santa Catarina - UFSC \\ Rod. SC 404, km 03 - Itacorubi \\ 88040-900 - Florianópolis, SC
}

\begin{abstract}
RESUMO
Estudou-se a presença de parasitas e realizaram-se exames macroscópicos e histológicos em ostras (Crassostrea rhizophorae e Crassostrea gigas) cultivadas. Entre agosto de 2002 a maio de 2003, 30 indivíduos adultos de cada espécie foram coletados sazonalmente, totalizando 240 ostras. Os animais, provenientes de desova em laboratório, foram mantidos em lanternas de cultivo, em sistema suspenso tipo espinhel, com densidade de 40 ostras/andar. A cada coleta era registrada a mortalidade das ostras, a temperatura e a salinidade da água. A temperatura variou de 19 a $28,5^{\circ} \mathrm{C}$ e a salinidade, 31 a $35 \%$. A mortalidade foi de $48,3 \%$ para C. gigas e $70,8 \%$ para C. rhizophorae. A infestação pelo poliqueta Polydora websteri em C. gigas foi $100 \%$ durante todo o período e em C. rhizophorae, $100 \%$ em fevereiro e maio. O mal do pé foi observado em novembro (3,3\%) e maio $(23,3 \%)$ em $C$. gigas e maio $(6,6 \%) \mathrm{em}$ C. rhizophorae. As maiores prevalências do protozoário Nematopsis sp. foram de 70 e $60 \%$ em C. gigas e C. rhizophorae, respectivamente. O protozoário Trichodina sp. ocorreu em 1,6\% de C. rhizophorae, e larvas do cestóide Tylocephalum sp. foram observadas em 2,5\% de C. gigas. Nenhum dos parasitas encontrados foi associado à mortalidade das ostras.
\end{abstract}

Palavras-chave: ostra, Crassostrea, histopatologia, parasita

\begin{abstract}
Over a 10-month period, cultured oysters (Crassostrea rhizophorae and Crassostrea gigas) from Ponta do Sambaqui, Florianopolis, Santa Catarina State were evaluated microscopically and histologically for parasite infection. Thirty mature individuals of each species were examined each season, for a total of 240 oysters. The animals, which originated from laboratory spawning, were kept in culture lanterns suspended in long-line systems at a density of 40 oysters per floor. Mortality, water temperature (19 to $28.5{ }^{\circ} \mathrm{C}$ range) and salinity (31 to 35\%o) were recorded at each sampling. The total amount of dead oysters was $58(48.3 \%)$ to $\mathrm{C}$. gigas and $85(70.8 \%)$ to C. rhizophorae. All C. gigas individuals were infected with polydiariosis parasites (100\%) throughout the experimental period; whereas $100 \%$ infection of G. rhizophorae with polydiariosis was observed only in February and May. "Foot disease" was detected in 3.3\% of C. gigas oysters in November and in $23.3 \%$ of C. gigas and $6.6 \%$ of C. rhizophorae oysters in May. The highest incidences of Nematopsis infection were $70 \%$ and $60 \%$ in $\mathrm{C}$. gigas and $\mathrm{C}$. rhizophorae, respectively. Trichodina protozoa occurred in 1.6\% of G. rhizophorae individuals; while Tylocephalum cestoda larvae were found in $2.5 \%$ of $\mathrm{C}$. gigas individuals examined. Total mortality percentages of 48.3 in $\mathrm{C}$. gigas and 70.8 in $\mathrm{C}$. rhizophorae could not be associated with parasite infection.
\end{abstract}

Keywords: oyster, Crassostrea, histopathology, parasite

Recebido para publicação em 27 de fevereiro de 2004

Recebido para publicação, após modificações, em 8 de outubro de 2004

E-mail: rachel@cca.ufsc.br 


\section{INTRODUÇ̃̃O}

Os moluscos bivalves marinhos podem ser afetados por muitos parasitas (Bower et al., 1994), que podem causar enfermidades e contribuir para diminuir as populações natural e, principalmente, cultivada. (Sindermann, 1970; Figueras e Villalba, 1988; Bower e Figueras, 1989). Informações sobre patógenos e seus efeitos sobre ostras e mexilhões vêm sendo acumuladas nos últimos 30 anos (Pavanelli et al., 2000). Segundo Villalba (2002), entre as enfermidades que afetam os moluscos bivalves marinhos no mundo, provocando perdas importantes, estão as causadas por protozoários, como Haplosporidium spp. e Perkinsus marinus em Crassostrea virginica, Mikrocytos mackini em Crassostrea gigas, Mikrocytos roughleyi em Saccostrea commercialis, Marteilia refringens em Ostrea edulis e Bonamia sp., Nematopsis sp. e Trichodina sp. em ostras dos gêneros Ostrea e Crassostrea.

Também estão bem documentados casos de parasitismo em ostras causados pelo anelídeo poliqueta Polydora sp., chegando a levar à mortalidade, principalmente de indivíduos jovens (Lauckner, 1983; Figueras e Villalba, 1988). Infestação por esse parasita, formando tubos $\mathrm{e}$ bolhas de lodo na concha de ostra C. gigas, cultivada no litoral de Santa Catarina, vem sendo registrada nos últimos anos (Ibbotson e Magalhães, 2002).

A enfermidade do pé ou mal do pé, causada pelo fungo Ostracobable implexa, tem sido responsável por elevada taxa de mortalidade em ostras do litoral da Holanda, França e Inglaterra (Figueras e Villalba, 1988). Em Santa Catarina, os problemas com essa enfermidade estão crescendo (Silveira Jr. et al., 2000).

Protozoários do gênero Nematopsis utilizam bivalves marinhos como hospedeiros intermediários e completam seu ciclo de vida no tubo digestivo de crustáceos (Azevedo e Cachola, 1992; Carballal et al., 2001). Esses organismos estão entre os mais importantes patógenos de bivalves, podendo ter diferentes estádios do seu ciclo de vida em diferentes tecidos do hospedeiro (Azevedo e Cachola, 1992). Nematopsis sp. foram registrados em Crassostrea rhizophorae da Baía de Todos os Santos-BA (Nascimento et al., 1986), em mexilhão Perna perna da Lagoa de Itaipu-RJ (Lima et al., 2001), em moluscos da região da Amazônia (Matos et al., 2001) e em berbigões Anomalocardia brasiliana de Florianópolis-SC (Magalhães et al., 2002).

Ciliados do gênero Trichodina parasitam ostras e outros bivalves marinhos. A patogenicidade desse parasita está associada à destruição dos filamentos branquiais e conseqüente perda de peso dos indivíduos (Bower et al., 1994; Figueras e Villalba, 1988). O parasita foi registrado em várias espécies de ostras da Europa e dos Estados Unidos (Bower et al., 1994; Boussaid et al., 1999).

Larvas de cestódeos, mais especificamente membros do gênero Tylocephalum, parasitam bivalves marinhos e ostras de várias partes do mundo, principalmente do gênero Crassostrea $\mathrm{e}$ ostras perlíferas. A penetração no hospedeiro ocorre através da brânquia ou epitélio do trato digestivo acompanhada de pronunciada reação celular dentro do tecido subepitelial e posterior encapsulação da larva (Sindermann, 1970; Lauckner, 1983). Metacestódeos de Tylocephalum sp. foram observados em ostras Crassostrea virginica (Cheng, 1967), C. gigas (Cheng, 1975), C. rhizophorae (Nascimento et al., 1986) e em algumas espécies do gênero Pinctada (Hine e Thorne, 2000).

O presente estudo teve como objetivo identificar parasitas nas ostras C. rhizophorae e C. gigas, cultivadas na Praia da Ponta do SambaquiFlorianópolis-SC.

\section{MATERIAL E MÉTODOS}

O trabalho foi realizado com ostras das espécies C. rhizophorae (Guilding, 1828) e C. gigas (Thunberg, 1797) provenientes de reprodução induzida e larvicultura no Laboratório de Moluscos Marinhos do Departamento de Aqüicultura da Universidade Federal de Santa Catarina. No mar, as ostras foram mantidas em lanternas em sistema de cultivo suspenso, tipo espinhel, localizado na Praia da Ponta do Sambaqui-Baía Norte da Ilha de Santa Catarina (2729'18"S e 4832'12"W).

Embora de idade semelhante no início do experimento, o tamanho mínimo das ostras foi de 
5,5 e 9,6cm para C. rhizophorae e C. gigas, respectivamente. Os moluscos foram acondicionados em quatro lanternas de cultivo (duas para cada espécie). Duas das lanternas eram experimentais e duas de reposição, para manter a densidade constante de 40 ostras/andar. As coletas ocorreram sazonalmente, no período de agosto de 2002 a maio de 2003, totalizando quatro coletas. Após cada coleta, as ostras foram acondicionadas em sacos plásticos etiquetados e transportadas em caixas isotérmicas até o laboratório, para biometria, análises macroscópicas e posteriores análises histológicas. A cada coleta, eram contadas as ostras mortas de cada espécie e medidas a temperatura e a salinidade da água do mar no local de cultivo. $\mathrm{O} n$ amostral foi de 30 indivíduos/espécie/coleta, perfazendo um total de 240 indivíduos analisados. O manejo das estruturas de cultivo para limpeza e retirada de incrustantes foi realizado mensalmente, durante todo o período do experimento.

A biometria das ostras foi feita com auxílio de paquímetro de precisão de $0,05 \mathrm{~mm}$, considerando o maior eixo como a altura, conforme proposto por Galtsoff (1964). Cada ostra, inicialmente examinada externamente, foi aberta com auxílio de um escalpelo inserido entre as valvas, para secção do músculo adutor e observações macroscópicas dos tecidos e da concha para detecção de parasitas.

Para as análises histológicas, foi retirada uma secção dos tecidos moles, em sentido diagonal na massa visceral, amostrando-se brânquia, manto, hepatopâncreas e gônadas de cada indivíduo, conforme recomendações de Howard e Smith (1983). Em seguida, os tecidos foram imersos em solução fixadora de Davidson por $48 \mathrm{~h}$ e retirada amostra para processamento histológico. Cortes de $7 \mu \mathrm{m}$ de espessura, feitos em micrótomo manual, foram corados pelos métodos hematoxilina e eosina. As lâminas histológicas, avaliadas em microscopia de luz para registro dos parasitas, foram fotomicrografadas e arquivadas no Laboratório de Moluscos Marinhos do Departamento de Aqüicultura da Universidade Federal de Santa Catarina.

Os dados obtidos foram submetidos ao teste $\mathrm{z}$ para comparação das proporções dos animais parasitados e não parasitados por Nematopsis sp. Utilizou-se como nível de significância $\alpha=0,05$.
$\mathrm{Z}_{\mathrm{c}}=\frac{\left[\hat{p}_{1}-\hat{p}_{2}\right]-\left[\frac{1}{2}\left(\frac{1}{n_{1}}+\frac{1}{n_{2}}\right)\right]}{\sqrt{\frac{\bar{p} \bar{q}}{n_{1}}+\frac{\bar{p} \bar{q}}{n_{2}}}}$, em que:

$\mathrm{z}_{\mathrm{c}}=\mathrm{z}$ calculado;

$\hat{p}_{1}=$ proporção amostral de Crassostrea gigas parasitada;

$\hat{p}_{2}=$ proporção amostral de Crassostrea

rhizophorae parasitada;

$n_{1}=$ tamanho da amostra de C. gigas;

$n_{2}=$ tamanho da amostra de $C$. rhizophorae;

$\bar{q}=1-\bar{p}$;

$\bar{p}=\frac{n_{1} \hat{p}_{1}+n_{2} \hat{p}_{2}}{n_{1}+n_{2}}$.

\section{RESULTADOS}

A temperatura da água do mar durante o período experimental variou de 19 (em maio) a $28,5^{\circ} \mathrm{C}$ (em fevereiro), e a salinidade, de 31 a $35 \%$ (em agosto e novembro), respectivamente. O tamanho das ostras variou de 5,5 a $7,8 \mathrm{~cm}$ para $C$. rhizophorae, e de 9,6 a $11,7 \mathrm{~cm}$, para $C$. gigas. Durante todo o período experimental, o número total de ostras mortas foi de 58 e 85 para $C$. gigas e C. rhizophorae, respectivamente. A mortalidade foi crescente para as duas espécies, sendo maior para a ostra nativa $C$. rhizophorae (Fig. 1).

A infestação pelo poliqueta espionídeo Polydora websteri em C. gigas foi elevada (100\%) durante todo o período. Em $C$. rhizophorae, o grau de infestação foi menor em agosto $(3,3 \%)$ e novembro $(0 \%)$, e maior $(100 \%)$ em fevereiro e maio de 2003. A formação de bolhas de lodo produzidas pelo poliqueta nas valvas internas das duas espécies estudadas também foi mais conspícua em fevereiro e maio.

As análises histológicas mostraram que a elevada infestação por $P$. websteri aparentemente não causou dano aos tecidos e órgãos dos animais e não interrompeu o processo de gametogênese. $\mathrm{O}$ aspecto macroscópico da polidiariose pode ser observado na Fig. 2. Em alguns raros casos, houve perfuração do tecido da ostra pelo tubo do poliqueto. Mesmo nesses casos, a ostra apresentou bom aspecto geral, macro e microscopicamente. 
A enfermidade do pé foi observada durante os meses de novembro $(3,3 \%)$ e maio $(23,3 \%)$ para C. gigas. Em C. rhizophorae, foi registrada somente no mês de maio, com $6,6 \%$ de prevalência. Essa enfermidade caracterizou-se por um nódulo de conchiolina na valva interna inferior da ostra na região do músculo adutor (Fig. 3).

As análises histológicas evidenciaram o parasitismo por protozoários dos gêneros Nematopsis e Trichodina, cestódeos do gênero Tylocephalum e larva de metazoário em estádio de reabsorção (Fig. 4).

A maior porcentagem de Nematopsis sp. em $C$. gigas e C. rhizophorae foi de 70\% (agosto) e $60 \%$ (fevereiro), respectivamente (Fig. 5). A análise estatística mostrou que a proporção da população parasitada foi igual para as duas espécies estudadas ao longo do experimento $(\mathrm{z}=$ $0,92)$. Esse parasita esteve presente em baixa intensidade na brânquia, lume do tubo digestivo, manto e hepatopâncreas. Apenas dois indivíduos apresentaram elevada intensidade de infecção na região do manto e epitélio do trato digestivo.

O ciliado Trichodina sp. foi observado no lume do tubo digestivo de $C$. rhizophorae e sua ocorrência foi de apenas $1,6 \%$.
Cestódeos do gênero Tylocephalum foram detectados entre os espaços do tubo digestivo e em torno do tubo digestivo de $C$. gigas, com prevalência de 2,5\%. Em um exemplar de $C$. rhizophorae foi registrada uma larva de metazoário encapsulada, em processo de reabsorção (Fig. 4H).

\section{DISCUSSÃO}

A infestação por $P$. websteri em $C$. gigas e $C$. rhizophorae causou alterações morfológicas nos tecidos dos animais, mas aparentemente não houve comprometimento da gametogênese. Ibbotson e Magalhães (2002), em Crassostrea gigas, registraram $100 \%$ de infestação por Polydora sp. nos meses de águas mais frias e concluíram que, apesar da alta taxa de prevalência, não houve dano aos órgãos ou tecidos dos indivíduos adultos, que apresentaram padrões normais no processo de formação dos gametas. Um levantamento ecológico realizado por Neptune et al. (2000), para avaliar a presença do poliqueta em ostras cultivadas na mesma região, registrou que $81,7 \%$ das ostras analisadas apresentavam túneis e/ou bolhas produzidas por $P$. websteri, e que o grau de infestação estava diretamente relacionado com o aumento da temperatura da água durante os meses de dezembro a janeiro, período de reprodução do poliqueta.

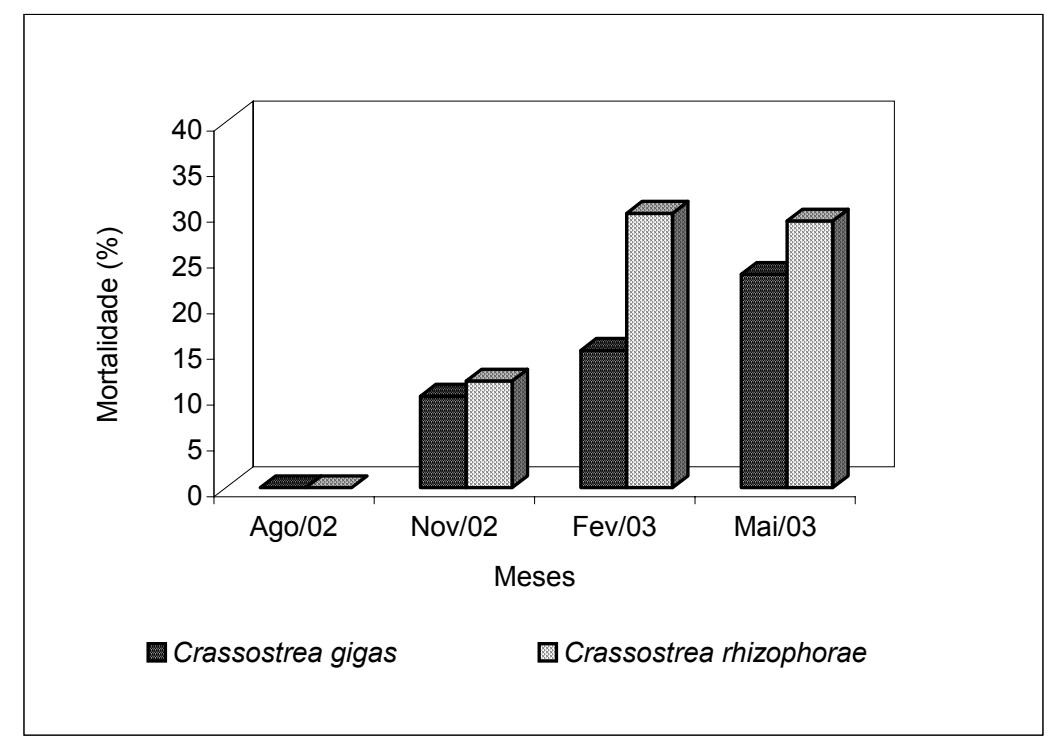

Figura 1. Mortalidade (\%) das ostras a cada amostragem. 

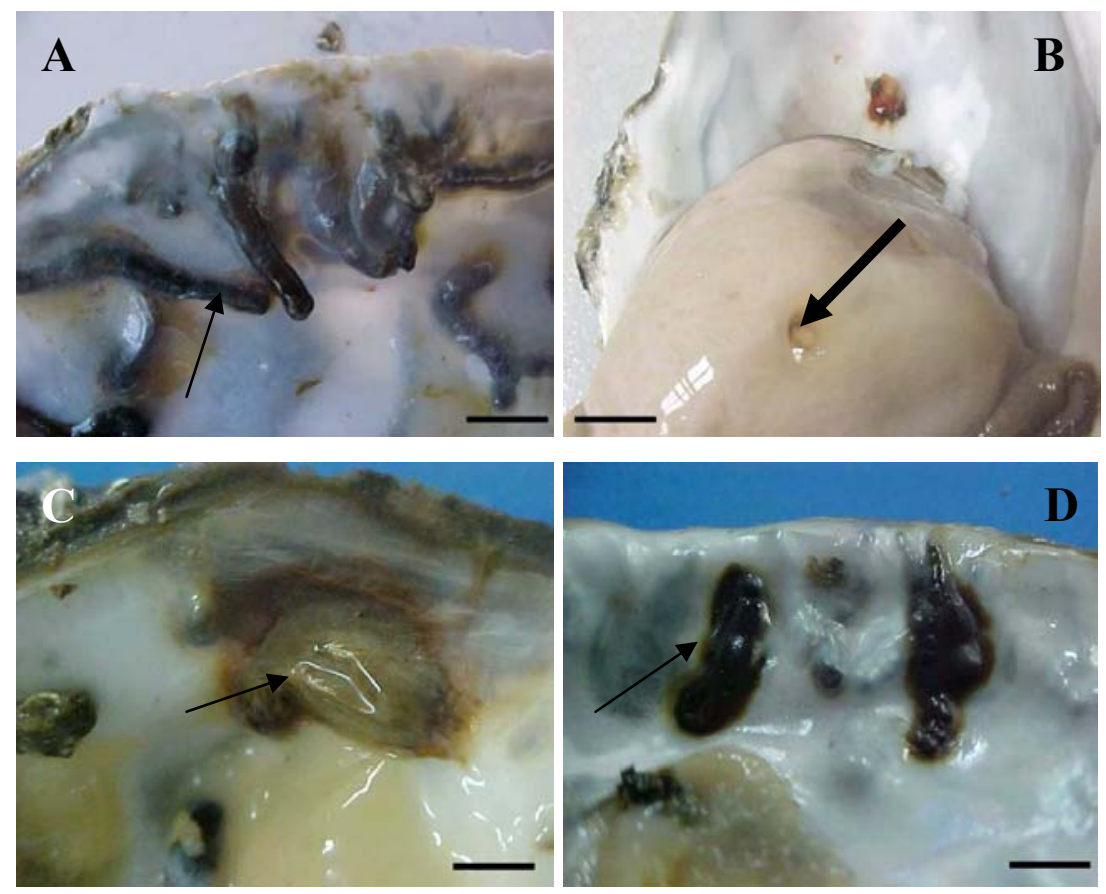

Figura 2. Polidiariose em ostras Crassostrea gigas (A) e Crassostrea rhizophorae (B), mostrando tubos (seta fina) e furo (seta larga) devido ao poliqueta Polydora sp.; bolhas de lodo em Crassostrea rhizophorae (C) e em Crassostrea gigas (D). Barra $=2 \mathrm{~cm}$.
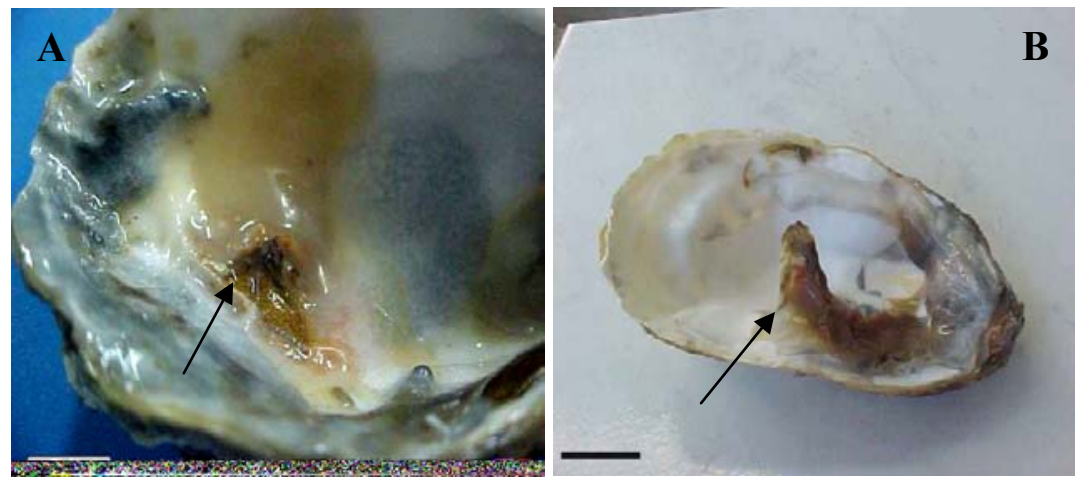

Figura 3. Mal do pé na área de insecção do músculo adutor de Crassostrea gigas. Em (A) estádio inicial, mostrando pequeno depósito de conchiolina em forma de nódulo e em (B), estádio avançado da enfermidade. Barra $=2 \mathrm{~cm}$. 

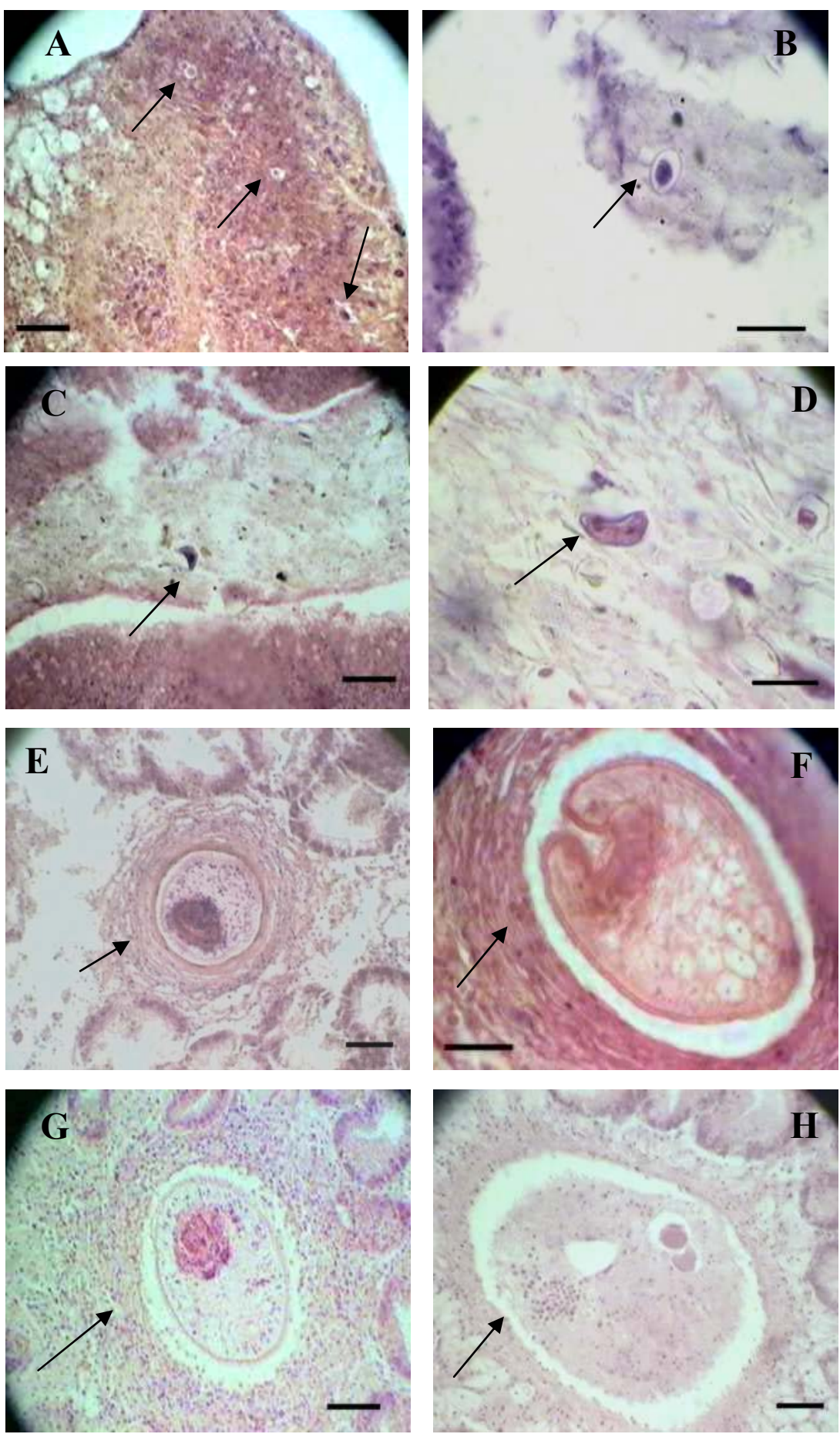

Figura 4. Fotomicrografias de parasitas em ostras Crassostrea gigas e Crassostrea rhizophorae. Nematopsis sp. no manto (A e B). Em C e D, Trichodina sp. no lume do tubo digestivo de Crassostrea rhizophorae. Em E, F e G, larvas de Tylocephalum em Crassostrea gigas (E - larva com encapsulamento fibroso denso, situada em torno do tubo digestivo; F-larva em fase metacestoda, em forma de coração, situada no tecido conjuntivo em torno do tubo digestivo; G - larva com encapsulamento menos denso situada entre os espaços do tubo digestivo). H - larva de metazoário em reabsorção em Crassostrea rhizophorae. Coloração HE. Barras: A, C, E, G e H $=50 \mu \mathrm{m}$ e B, D e F $=25 \mu \mathrm{m}$. 


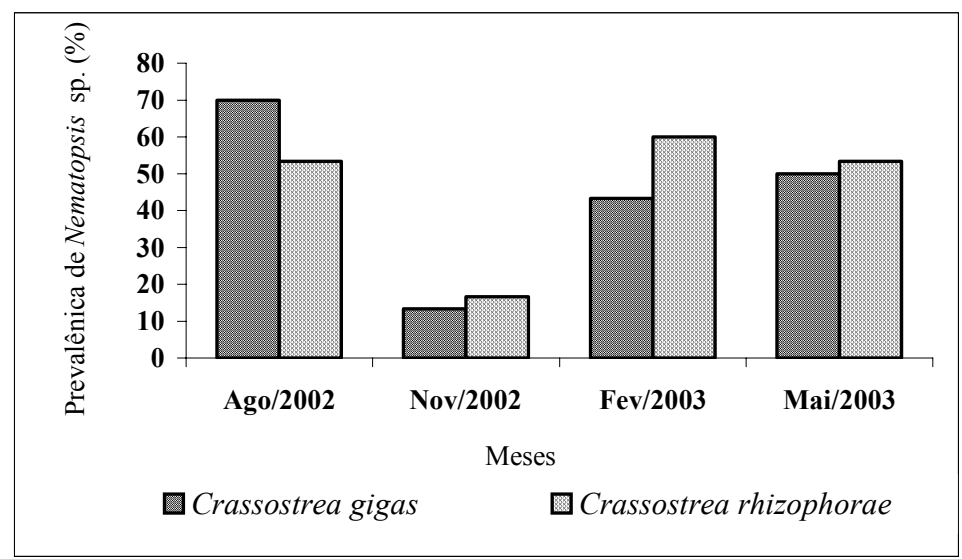

Figura 5. Prevalência (\%) de Nematopsis sp. nos meses amostrados.

A elevada prevalência de $P$. websteri em $C$. gigas durante todo o período experimental sugere que a população desse anelídeo espionídeo está gradualmente aumentando nas áreas de cultivo, nos últimos anos. Nel et al. (1996), ao avaliarem a infestação por Polydora em C. gigas no sul da África, registraram maior infestação do verme durante o verão, com um valor alto também no início do outono. Ressaltaram que, apesar do elevado índice de infestação, esses indivíduos estariam aptos a se reproduzir logo que a temperatura da água voltasse a aumentar (primavera). Caceres-Martinez et al., (1998), em cultivos de $C$. gigas na Baixa Califórnia, verificaram que o verme formava canais em forma de $\mathrm{U}$ e bolhas de lodo, abundantes quando a temperatura da água estava mais alta. No entanto, ressaltaram que a maior prevalência de infestação do parasita poderia ser atribuída ao maior tempo de exposição do hospedeiro, uma vez que eles ficaram permanentemente submersos e próximos ao substrato. Ainda, segundo os autores, Polydora sp. não foi responsável pela mortalidade das ostras, pois entre os indivíduos mortos, apenas dois apresentavam bolhas de lodo produzidas pelo parasita.

Neste trabalho, a formação de tubos por Polydora em C. rhizophorae foi menor do que em C. gigas: 3,3\% em agosto e ausente em novembro. Isso sugere ser essa última espécie mais susceptível ao ataque desse parasita. A maior ocorrência de Polydora e/ou bolhas de lodo na ostra nativa em fevereiro e maio pode estar relacionada com temperaturas mais altas da água, pois, segundo Lauckner (1983), os parâmetros de temperatura e salinidade têm sido reportados como fatores primários na determinação da abundância de Polydora. $P$. websteri foi provavelmente o principal produtor de bolhas em 69,4\% das ostras $C$. gigas da região de Mahurangi Harbour- Nova Zelândia (Handley e Bergquist, 1997). A presença de bolhas ou vesículas na cavidade interna do manto faz com que as ostras incrementem a deposição de concha para repor o volume e a forma interna da concha (Handley, 1998). As bolhas de lodo afetam o aspecto, o sabor e o valor de mercado desses moluscos. Além disso, os elevados índices de infestação por Polydora podem diminuir a taxa de crescimento e o índice de condição (IC) dos animais infestados em relação aos não infestados (Ambariyanto, 1991).

A incidência do mal do pé, tanto em $C$. gigas quanto em $C$. rhizophorae nos meses de novembro e maio, coincidiu com o aumento de temperatura da água. A ocorrência da enfermidade está crescendo, há alguns anos, no estado de Santa Catarina. Em estudos realizados com ostras $C$. gigas provenientes de cultivos do litoral catarinense, foi registrada variação de 2,0 a 9,5\% na ocorrência dessa doença (Silveira Jr. et al., 2000). O fungo causador dessa enfermidade é desconhecido. Um fungo filamentoso semelhante (Ostracobable implexa) que se desenvolve bem em temperaturas em torno de $30^{\circ} \mathrm{C}$ tem sido responsável pela elevada taxa de mortalidade em 
moluscos na Holanda, França e Inglaterra (Figueras e Villalba, 1988).

A presença de Nematopsis sp. em C. gigas e $C$. rhizophorae aparentemente não foi responsável pela mortalidade das ostras durante o período experimental, uma vez que o grau de infecção observado nas duas espécies foi baixo, com exceção de apenas dois indivíduos. Eles apresentaram maior ocorrência do parasita no manto e no epitélio do trato digestivo, acompanhada de reação inflamatória. No mês de novembro houve diminuição da carga parasitária para as duas espécies, no entanto não foi possível associar essa diminuição ao aumento de temperatura da água nesse período, tendo em vista que outros fatores podem ter influenciado nesse resultado. Nascimento et al. (1986) observaram a ocorrência de $100 \%$ de Nematopsis sp. em C. rhizophorae da Baía de Todos os Santos - BA e concluíram que o parasita não foi o fator determinante para a mortalidade das ostras.

No molusco Cerastoderma edule da região sul de Portugal, Nematopsis sp. foi observado no tecido conectivo da brânquia, chegando a parasitar $82 \%$ dos animais analisados (Azevedo e Cachola, 1992). A infecção mais intensa foi observada nos animais maiores, causando completa destruição das células das brânquias e elevada taxa de mortalidade. Na costa da Galícia, a mesma espécie citada apresentou $76 \%$ de indivíduos com o protozoário. No entanto, ele não causou a morte dos moluscos e nenhum dano patológico significante (Carballal et al., 2001). A patogenicidade desse parasita sobre o hospedeiro ainda é inconclusiva (Lauckner, 1983; Bower e Figueras, 1989).

O ciliado Trichodina sp., observado somente em C. rhizophorae, parece não ter causado dano ao hospedeiro, apesar de, geralmente, ocorrer próximo ao manto, palpos labiais ou na superfície das brânquias. Neste estudo foi encontrado livre no lume do tubo digestivo. Alta infecção por Trichodina sp. em Crassostrea angulata na França foi relacionada com destruição dos filamentos branquiais e perda de peso dos animais (Bower et al., 1994). C. gigas, altamente infectada pelo parasita, também apresentou processo inflamatório nas brânquias e alterações do epitélio (Boussaid et al., 1999). Ainda, segundo esses autores, embora existam controvérsias sobre a patogenicidade de Trichodina, acredita-se que o parasita seja responsável por alta mortalidade em muitos animais marinhos.

A presença de larvas de Tylocephalum sp. aparentemente não causou dano aos tecidos e órgãos de $C$. gigas, pois foi possível a determinação do sexo e fases do ciclo reprodutivo dos animais infectados. Esse parasita foi observado tanto em machos como em fêmeas. As larvas de Tylocephalum, alojadas entre os espaços do hepatopâncreas, apresentaram uma cápsula fibrosa menos densa do que aquelas encistadas no conjuntivo ao seu redor. A formação dessa cápsula fibrosa e a infiltração de hemócitos são uma resposta do hospedeiro contra o parasita. Segundo Lauckner (1983), maior ou menor intensidade de encapsulamento pode estar relacionada com o tipo e a quantidade de células presentes no local. Segundo Cheng (1975), metacestódeos encistados em $C$. gigas apresentaram cápsula menos densa e semelhante às que foram observadas em $C$. virginica. A resposta de $C$. gigas à infecção por metacestódeo de Tylocephalum parece ser menos intensa que em outros bivalves (Cheng, 1975; Figueras e Villalba, 1988). Segundo Lauckner (1983), em ostras do gênero Pinctada, a fibrose induzida é relativamente menor, pois acredita-se que elas sejam hospedeiras intermediárias normais do parasita. No entanto, ressalta que a patologia da infecção desse parasita pode variar com a espécie hospedeira.

A larva de metazoário, em estádio de reabsorção, observada em $C$. rhizophorae no mês de fevereiro, pode provavelmente ser de Tylocephalum. Larvas de metacestódeos em reabsorção foram registradas em $C$. virginica por Cheng (1967). O autor concluiu que, provavelmente, a espécie não era o hospedeiro intermediário normal do parasita.

No presente estudo, foram registradas novas ocorrências de parasitas em ostras do litoral brasileiro. Todavia, nenhum dos parasitas encontrados pôde ser associado à mortalidade registrada durante o experimento.

Apesar da alta prevalência de alguns parasitas encontrados, como o poliqueta Polydora e o protozoário Nematopsis, não houve comprometimento de atividades vitais das ostras, 
seja pela quantidade de parasitas por hospedeiro e /ou a relação entre ambos.

\section{REFERÊNCIAS BIBLIOGRÁFICAS}

AMBARIYANTO, S.R. The infestation of Mytilus edulis Linnaeus by Polydora ciliata (Johnston) in the Conwy Estuary, North Wales. J. Moll. Stud., v.57, p.413-424, 1991.

AZEVEDO, C.; CACHOLA, R. Fine structure of the apicomplexa oocyst of Nematopsis sp. of two marine bivalve molluscs. Dis. Aquat. Org., v.14, p.69-73, 1992.

BOUSSAID, B.; GRIPPARIT, J.L.; RENAULT, T. et al. Trichodina sp. infestation of Crassostrea gigas oyster gills in Brittany, France. $J$. Invertebr. Pathol., v.73, p.339-342, 1999.

BOWER, S.M.; FIGUERAS, A.J. Infectious diseases of mussels, especially pertaining to mussel transplantation. World Aquacult. Rev., v.20, p.89-93, 1989.

BOWER, S.M.; MCGLADDERY, S.E.; PRICE, I.M. Synopsis of infectious diseases and parasites of commercially exploited Shellfish. Annu. Rev. Fish. Dis., v.4, p.1-199, 1994.

CACERES-MARTINEZ, J.; DE OCA, P.M.M.; VASQUEZ-YEOMANS, R. Polydora sp. infestation and health of the Pacific oyster Crassostrea gigas cultured in Baja California, NW México. J. Shellf. Res., v.17, p.259-264, 1998.

CARBALLAL, M.J.; IGLESIAS, D.; SANTAMARINA, J. et al. Parasites and pathologic conditions of the cockle Cerastoderma edule populations of the Coast of Galicia (NW Spain). J. Invertebr. Pathol., v.78, p.87-97, 2001.

CHENG, T.C. New geographic records for Tylocephalum metacestodes. J. Inverterbr. Pathol., v.26, p.395-396, 1975.

CHENG, T.C. Parasites of commercially important marine molluscs. In: RUSSEL, F. C. (Ed). Advances in marine biology. New York: Academic, 1967. cap. 6, p.199-261.

FIGUERAS, A.J.; villalba, A. Patología de moluscos. In: MONTEROS J.E.; LABARTA, U. (Eds.). Patología en acuicultura. Madrid: Mundi-Prensa Libros, 1988. cap. 4, p.327-376.
GALTSOFF, P.S. The American oyster Crassostrea virginica. Fish. Bull., v.64, p.1-480, 1964.

HANDLEY, S.J. Power to the oyster: do spionidinduced shell blisters affect condition in subtidal oysters?. J. Shellf. Res., v.17, p.1093-1099, 1998.

HANDLEY, S.J.; BERGQUIST, P.R. Spionid polychaete infestations of intertidal pacific oysters Crassostrea gigas (Thunberg), Mahurangi Harbour, northern New Zealand. Aquaculture, v.153, p.191-205, 1997.

HINE, P.M.; THORNE, T. A survey of some parasites and diseases of several species of bivalve mollusc in northern Western Australia. Dis. Aquat. Org., v.40, p.67-68, 2000.

HOWARD, D.W.; SMITH, C.S. Histological techniques for marine bivalve mollusks. Woods Hole, Massachussetts: NOAA Technical Memorandum, 1983. 97p.

IBBOTSON, D.P.; MAGALHÃES, A.R.M. Polidiariose em Crassostrea gigas cultivadas na Praia da Ponta do Sambaqui, Florianópolis, SC, Brasil. In: ENCONTRO BRASILEIRO DE PATOLOGISTAS DE ORGANISMOS AQUÁTICOS, 7.; ENCONTRO LATINO AMERICANO DE PATOLOGISTAS DE ORGANISMOS AQUÁTICOS, 3., 2002, Foz do Iguaçu. Anais... Foz do Iguaçu: UEM, 2002. p.192.

LAUCKNER, G. Diseases of Mollusca:Bivalvia. In: KINNE, O (Ed.). Diseases of marine animals. Hamburg: Biologische Anstalt Helgoland, 1983. cap. 13, p.477-879.

LIMA, F.C.; ABREU, M.G.; MESQUITA, E.F.M. Monitoramento histopatológico de mexilhão Perna perna da Lagoa de Itaipu, Niterói, RJ. Arq. Bras. Med. Vet. Zootec., v.53, p.203-206, 2001.

MAGALHÃES, A.R.M.; MATOS, E.; AZEVEDO, C. Dados ultraestruturais do oocisto de Nematopsis sp. (Phylum Apicomplexa) parasita do berbigão, Anomalocardia brasiliana Gmelin, 1791 (Mollusca, Bivalvia, Veneridae) da região de Florianópolis, Santa Catarina. In: ENCONTRO BRASILEIRO DE PATOLOGISTAS DE ORGANISMOS AQUÁTICOS, 7.; ENCONTRO LATINO AMERICANO DE PATOLOGISTAS DE ORGANISMOS AQUÁTICOS, 3., 2002, Foz do 
Iguaçu. Anais... Foz do Iguaçu: UEM, 2002. p.191.

MATOS, E.; MATOS, P.; SANTOS, M.M.S. et al. Ação de protozoários parasitas em moluscos da Região Amazônica: Nematopsis sp. In: ENCONTRO BRASILEIRO DE MALACOLOGIA, $\quad 17$; $\quad$ SIMPÓSIO NORDESTINO DE CULTIVO DE MOLUSCOS BIVALVES, 1., 2001, Recife, Anais... Recife: UFPE, 2001. p.78.

NASCIMENTO, I.A.; SMITH, D.H.; KERN II, F. et al. Pathological findings in Crassostrea rhizophorae from Todos os Santos Bay, Bahia, Brazil. J. Invertebr. Pathol., v.47, p.340-349, 1986.

NEL, R.; COETZEE, P.S.; NIEKERK, G.V. The evaluation of two treatments to reduce mud worm (Polydora hoplura Claparede) infestation in commercially reared oysters (Crassostrea gigas Thunberg). Aquacultura, v.141, p.31-39, 1996.

NEPTUNE, Y.M.B.; POLI, C.R.; FERREIRA, J.F. Dados ecológicos sobre poliqueta Polydora websteri (Hartman)(Fam. Spionidae) em cultivo da ostra do Pacífico Crassostrea gigas (Thunberg, 1793) em Florianópolis. In: ENCONTRO BRASILEIRO DE PATOLOGISTAS DE ORGANISMOS AQUÁTICOS, 6.; ENCONTRO LATINO AMERICANO DE PATOLOGISTAS DE ORGANISMOS AQUÁTICOS, 2., 2000,
Florianópolis. Anais... Florianópolis: UFSC, 2000. p.31.

PAVANELLI, G.C.; EIRAS, J.E.; TAKEMOTO, R.M. et al. Sanidade de peixes, rãs, cristáceos e moluscos. In: VALENTI, W.C.; POLI, C.R.; PEREIRA, J.A. et al. (Eds.). Aqüicultura no Brasil: bases para um desenvolvimento sustentável. Brasília: CNPq/Ministério da Ciência e Tecnologia, 2000. Cap. 6, p.197-245.

SILVEIRA JUNIOR, N.; MAGALHÃES, A.R.M.; BRAGA, F.E. Evolução e sintomatologia da "doença do péc" em ostras do Pacífico (Crassostrea gigas) cultivadas em Florianópolis/SC-Brasil. In: ENCONTRO BRASILEIRO DE PATOLOGISTAS DE ORGANISMOS AQUÁTICOS, 6.; ENCONTRO LATINO AMERICANO DE PATOLOGISTAS DE ORGANISMOS AQUÁTICOS, 2., 2000, Florianópolis. Anais... Florianópolis:UFSC, 2000. p.26.

SINDERMANN, C.J. Principal diseases of marine fish and shellfish. New York: Academic, 1970. 369p.

VILLALBA, A. Patología de moluscos bivalvos. In: ENCONTRO BRASILEIRO DE PATOLOGISTAS DE ORGANISMOS AQUÁTICOS, 7.; ENCONTRO LATINO AMERICANO DE PATOLOGISTAS DE ORGANISMOS AQUÁTICOS, 3., 2002, Foz do Iguaçu. Anais... Foz do Iguaçu: UEM, 2002. p.201. 